\title{
SIMULATION OF BREED AND BURN FUEL CYCLE OPERATION OF MOLTEN SALT REACTOR IN BATCH-WISE REFUELING MODE
}

\author{
Valeria Raffuzzi $^{1}$ and Jiri Krepel ${ }^{1}$ \\ ${ }^{1}$ Paul Scherrer Institute \\ Forschungsstrasse 111, 5232 Villigen, Switzerland \\ valeria.raffuzzi@psi.ch,jiri.krepel@psi.ch
}

\begin{abstract}
The Molten Salt Reactor (MSR) is one of the most revolutionary Gen-IV reactors and it can be operated, especially with chloride salts, in the so-called breed and burn fuel cycle. In this type of fuel cycle the fissile isotopes from spent fuel do not need to be reprocessed, because the excess bred fuel covers the losses. The liquid phase of the MSR fuel assures its instant homogenization, and the reactor can be operated with batch-wise refueling thus reaching an equilibrium state. At the same time, the active core of the chloride fast MSR needs to be bulky to limit neutron leakage. In this study, the code Serpent 2 was coupled to the Python script BBP to simulate batch-wise operation of the breed and burn MSR fuel cycle. The script, previously developed for solid assemblies shuffling, was modified to simulate fuel homogenization after fertile material addition. Several fuel salts and fission products removal strategies were simulated and their impact was analyzed. Similarly, the influence of blanket volume was assessed in a two-fluid core layout. The results showed that the reactivity initially grows during the irradiation period and later decreases. The blanket has a large impact on the performance and it can be used to further increase the fuel burnup or to shrink the active core size. The breed and burn fuel cycle in MSR can reach high fuel utilization without fuel reprocessing and a multi-fluid layout can help to decrease the core size.
\end{abstract}

KEYWORDS: Molten Salt Reactor, Breed and Burn, Equilibrium cycle

\section{INTRODUCTION}

The Molten Salt Reactor (MSR) was selected as one of the six advanced Generation IV nuclear systems by the Generation IV International Forum. MSRs with liquid fuel look particularly promising due to their many advantages, such as the absence of integrity issues related to solid fuel and the possibility of online fuel reprocessing. MSRs could also be operated in Breed and Burn (B\&B) mode. In this type of fuel cycle, the fresh fuel feed is totally fertile and all the fissile material to be burnt is bred inside the reactor. B\&B operation mode offers high fuel utilization even in an open once-through cycle. As a consequence the most expensive steps of the fuel cycle, fuel enrichment and reprocessing, are no longer necessary. To be able to sustain itself, a B\&B reactor must benefit from an excellent neutron economy. For this reason this fuel cycle operation is mainly applicable to fast reactors. In this work, a conceptual MSR design was simulated to optimize its neutronic performances. The system is a "fuel pool", externally cooled fast reactor fueled with a homogeneous liquid salt; single and multi-fluids configurations were modeled. First, the neutronic feasibility of the concept was assessed. Then, its geometry was optimized. 


\section{SIMULATION TOOLS AND METHODOLOGY}

MSR can be operated in batches, i.e. in repetitive cycles. After several cycles in which the reactor parameters are unchanged, the reactor converges to an equilibrium state. At equilibrium the core reactivity, fuel composition and neutron flux distribution are constant from cycle to cycle. Therefore, the equilibrium condition is often used in order to compare and evaluate the performances of different reactors [1].

The tool used to bring the system to equilibrium is called Batch Burnup Procedure (BBP). It is a Python routine developed at Paul Scherrer Institute to simulate solid assemblies refueling [2], and subsequently adapted to include the application to homogeneous liquid fuels. The BBP script internally calls the Monte Carlo code Serpent 2 to run neutronic calculations. At the end of each cycle, BBP automates refueling by manipulating Serpent outputs and creating the material input for the following cycle. The routine is repeated until the system converges to equilibrium.

\section{FEASIBILITY STUDY}

Previous studies, like [3] and [4], have demonstrated the feasibility of the aforementioned MSR concept with continuous refueling. In this study batch-wise refueling, which is more practical from an application perspective, could be implemented through the BBP routine. One year long cycles were adopted. The feasibility of the B\&B fuel cycle operation is assessed in an infinite model, which is zero-dimensional in the case of a homogeneous medium. With an infinitely periodic system, the neutronic performances are the best possible due to the absence of leakage, which is particularly relevant in molten salts. At equilibrium, a $\mathrm{B} \& \mathrm{~B}$ reactor converges to its natural iso-breeding material composition. In this case, feasibility is confirmed if there is positive reactivity after convergence, i.e. the net neutron production is positive and the reactor can sustain itself as an isobreeder [5]. $k_{\text {infinite }}$ is expressed as a function of the equilibrium burnup. Since the fuel salt is assumed to be homogeneously mixed, burnup is also a homogeneous property of the fuel medium and is related to the average between the residence times of each batch of fuel.

Different fuel salts were studied; their compositions are listed in Table I. A Uranium cycle, a Thorium cycle and a mixed one were simulated and compared. Only Chloride salts were considered because previous studies proved them to perform neutronically better than Fluorides [6]. More specifically, ${ }^{37} \mathrm{Cl}$ enrichment is needed, due to its favorable neutronic properties compared to ${ }^{35} \mathrm{Cl}$. In this work, Chlorine is composed $100 \%$ by ${ }^{37} \mathrm{Cl}$. Nevertheless, other issues connected to the use of Chloride salts still have to be solved, like material corrosion related issues and establishing an economical enrichment method.

Table I. Fuel salt compositions: Fuel salts included in the B\&B feasibility study [6].

\begin{tabular}{|c|c|c|c|}
\hline Salt composition & Atomic fractions [\%] & Density $\left[\frac{\mathrm{g}}{\mathrm{cm}^{3}}\right]$ & Melting Temperature $\left[{ }^{\circ} \mathrm{C}\right]$ \\
\hline $\mathrm{NaCl}-\mathrm{UCl}_{3}$ & $60-40$ & 3.64 & 590 \\
\hline $\mathrm{NaCl}-\mathrm{ThCl}_{4}-\mathrm{UCl}_{3}$ & $50-25-25$ & 3.16 & $\approx 500$ \\
\hline $\mathrm{NaCl}-\mathrm{ThCl}_{4}$ & $55-45$ & 3.15 & 375 \\
\hline
\end{tabular}

Non-soluble fission and decay products removal was included. Most of the fission products dissolve in the fuel salt. However, some elements are non-soluble and could be physically separated from the salt, for example by Helium bubbling. These products are noble gases and volatile products such as $\mathrm{He}, \mathrm{Ne}, \mathrm{Ar}$ and $\mathrm{Xe}$, and some metals and semi-metals as $\mathrm{Nb}, \mathrm{Mo}, \mathrm{Tc}, \mathrm{Ru}, \mathrm{Te}$, etc [7]. Two different products removal modes were simulated. First batch-wise removal, applied at the end of each cycle through the BBP routine. In a second place, continuous removal was implemented through an extension of Serpent2 appositely created 
for reprocessing in liquid fuel systems [7]. In this version, a fictitious decay term is added to the depletion equation: the removal is exponential, and depends on a time constant chosen by the user. The nominal time constant used in this work is $\tau=\frac{1}{30 s}$. Figure 1 shows the performance of the different fuel salts proposed.

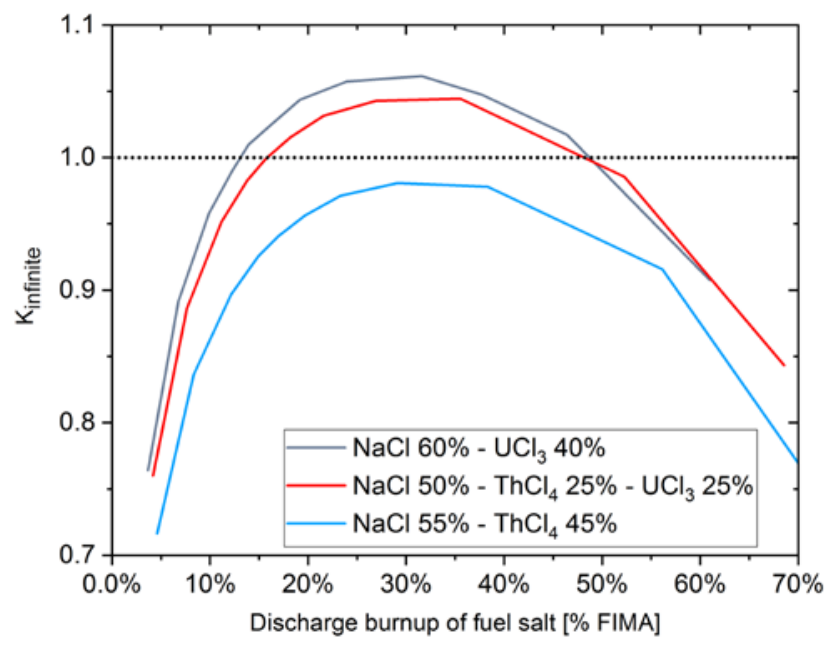

Figure 1. Comparison between fuel salts. Neutron multiplication factor as a function of average burnup for different salts, with continuous non-soluble products removal applied.

Uranium salt is the best performing, and it could be operated with an average fuel burnup included in the range between 13\% and 49\% Fissions per Initial Metal Atom (FIMA). Outside of this range, when burnup is too low, sufficient breeding of fissile material to sustain the chain reaction cannot be achieved; if it's too high, however, fission products poisoning creates a strong negative reactivity insertion. Therefore, the maximum occurs in a mid-burnup interval, i.e. between $25 \%$ and $35 \%$ FIMA. The mixed Uranium and Thorium cycle shows positive reactivity in the burnup range from $15 \%$ to $47 \%$. The pure Thorium salt, however, isn't self-sustainable with B\&B operation.

Fig. 2 shows that removal of non-soluble fission and decay products, which can be strong parasitic absorbers, is strictly necessary. Continuous removal is the best option. In practical terms, continuous removal can be achieved via Helium bubbling in the core: Helium bubbles are sprayed in the salt, trap the undissolved products and are eventually collected in the off-gas system. The difference between each removal case and the others is more evident at high burnup, when soluble products generated by decay or transmutation of the un-removed non-soluble fission products sit in the core for a longer time.

A sensitivity study on the cycle length as well as the time removal constant $\tau$ was carried out. The equilibrium average masses of non-soluble fission products present in the core were compared for the different cases studied. They are summarized in Table II. Continuous products removal is consistently more efficient than batch-wise removal, even after decreasing significantly the time removal constant.

From Table II it can be noticed that, in the batch-wise removal case, the mass of non-soluble fission products in the core is linearly proportional to the cycle length. Using this relation it can be calculated that, in order to reach the performances of the nominal continuous removal case $(\tau=1 / 30 \mathrm{~s})$ with batch-wise removal, the cycle should be 26 seconds long. This is, of course, unpractical and unrealistic. Considering also that $k_{\text {infinite }}$ and the maximum achievable burnup are not sensitive to small $\tau$ variations, continuous removal with $\tau=1 / 30$ s is considered to be a good modeling of the best realistic removal strategy. It must be 
observed that in this study, $100 \%$ fission products removal efficiency was assumed. The set-up implemented in the Serpent model includes 2000 neutrons per cycle and 300 active and 30 inactive cycles.

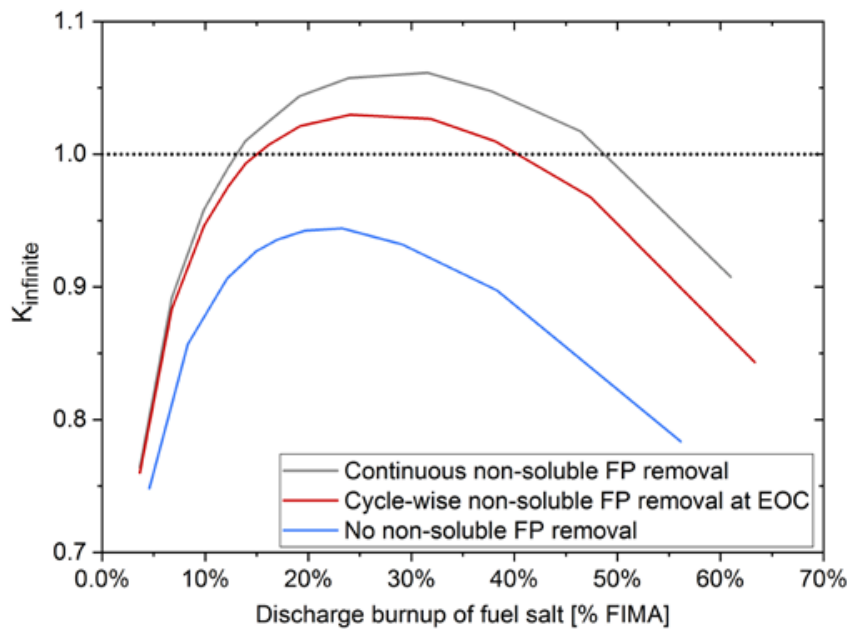

Figure 2. Comparison between non-soluble products removal strategies. Neutron multiplication factor as a function of average burnup. The fuel used is the previously introduced Uranium salt.

Table II. Comparison of seven removal modalities: Comparison between neutronic performances of situations with different non-soluble products removal strategies (other than removal by refueling).

\begin{tabular}{|c|c|c|c|c|}
\hline \multicolumn{2}{|c|}{ Non-sol. FP removal mode } & Non-soluble FP mass in the core $\left[\mathbf{g} / \mathbf{c m}^{3}\right]$ & Max $\boldsymbol{k}_{\text {inf }}$ & Max BU [FIMA] \\
\hline \multicolumn{2}{|c|}{ No removal } & $2.12 \mathrm{E}-01$ & 0.94411 & $19 \%$ \\
\hline \multirow{2}{*}{$\begin{array}{c}\text { Cycle-wise } \\
\text { removal }\end{array}$} & 2 years & $8.96 \mathrm{E}-03$ & 1.02631 & $40 \%$ \\
\cline { 2 - 5 } & 1 year & $4.40 \mathrm{E}-03$ & 1.02979 & $40 \%$ \\
\cline { 2 - 5 } & $1 / 2$ years & $2.18 \mathrm{E}-03$ & 1.03222 & $40 \%$ \\
\hline $\begin{array}{c}\text { Continuous } \\
\text { removal }\end{array}$ & $\tau=1 / 30000 s$ & $8.90 \mathrm{E}-06$ & 1.05310 & $46 \%$ \\
\cline { 2 - 5 } & $\tau=1 / 300 s$ & $5.18 \mathrm{E}-08$ & 1.05653 & $48 \%$ \\
\cline { 2 - 5 } & $\tau=1 / 30 s$ & $3.68 \mathrm{E}-09$ & 1.06142 & $49 \%$ \\
\hline
\end{tabular}

\section{FINITE CORE OPTIMIZATION}

Once the feasibility of B\&B fuel cycle mode for an infinite core was confirmed, a finite geometry was simulated. MSRs fueled by Chlorides need to be extremely bulky: ${ }^{37} \mathrm{Cl}$ has a low scattering cross section and high atomic mass compared to moderator materials. As a consequence, neutron leakage outside of the reactor is a big loss which must be compensated for. The selected geometry is a cylinder with proportions $\frac{D}{H}=0.92$, which have been proven to be optimal by diffusion theory. The molten salt is contained into a $2.5 \mathrm{~cm}$ thick Hastelloy vessel. The set-up implemented in the Serpent model includes 2000 neutrons per cycle and 300 active and 30 inactive cycles.

\subsection{Minimum critical dimensions}

In this section, criticality was searched for in the described geometry, varying the core diameter. A bare reactor was investigated, as well as a reactor surrounded by a one meter thick reflector. Two reflector materials were considered, Lead and Hastelloy. According to Table III, introducing a reflector allows a 
smaller fuel inventory. In particular, Lead is more efficient than Hastelloy. However, even the smallest core volume found, $305 \mathrm{~m}^{3}$, would be extremely large for practical application. Therefore, a geometry optimization is needed. The proposal of a $200 \mathrm{~m}^{3} \mathrm{~B} \& \mathrm{~B}$ MSR design can be seen in [8].

Table III. Finite core criticality: Smallest critical geometry for a cylindrical reactor with and without reflector.

\begin{tabular}{|c|c|c|c|}
\cline { 2 - 4 } \multicolumn{1}{c|}{} & Optimal BU [FIMA] & Core Diameter [m] & Core Volume $\left[\mathbf{m}^{\mathbf{3}}\right]$ \\
\hline Bare Reactor & $32 \%$ & 9 & 527 \\
\hline With Hastelloy Reflector & $32 \%$ & 8.2 & 398 \\
\hline With Lead Reflector & $32 \%$ & 7.5 & 305 \\
\hline
\end{tabular}

\subsection{Two-fluid reactor}

A fertile salt blanket can be added between the core and the Lead reflector, to make use of the neutrons leaking outside of the core. Fissile material is bred inside the blanket during irradiation; at the end of each cycle, the core is refilled with the salt from the blanket and the blanket is refilled with fresh fertile feed. The blanket can effectively limit neutron leakage when its equilibrium reactivity is negative, in which case the radial neutron flux would decay exponentially from the inside towards the outside. Additionally, the optimal blanket burnup is low, to prevent the blanket from acting like a burner, depleting the fissile material bred and accumulating poisonous fission products.

Three parameters were investigated: the core volume, the blanket volume and the total salt burnup. The core volumes simulated are $100 \mathrm{~m}^{3}, 200 \mathrm{~m}^{3}$ and $300 \mathrm{~m}^{3}$; the blanket volumes $50 \mathrm{~m}^{3}, 100 \mathrm{~m}^{3}, 150 \mathrm{~m}^{3}$ and $250 \mathrm{~m}^{3}$. Fig. 3 is an in-scale representation of the geometries considered. The system was optimized in terms of $k_{\text {effective }}$ and of the total dimensions.
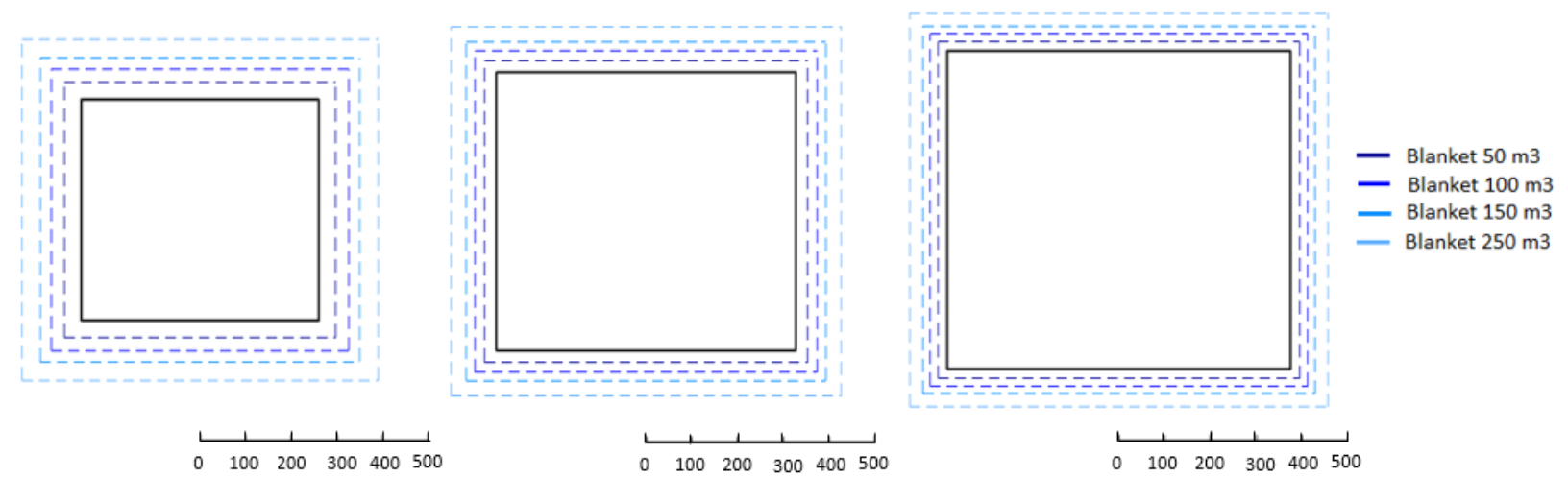

Figure 3. Core and blanket volumes. On scale representation of the core dimensions with different blankets. The core volume is, from left to right, $100 \mathrm{~m}^{3}, 200 \mathrm{~m}^{3}$ and $300 \mathrm{~m}^{3}$. The unit of the scale is $\mathrm{cm}$.

Fig. 4 shows the dependence of $k_{\text {effective }}$ on the outer radius of the blanket and on the burnup. Three total burnups were simulated: $22 \%, 33 \%$ and $44 \%$. Among these values, the best equilibrium composition is the one burnt up to $33 \%$ FIMA. Additionally, a more detailed analysis on the impact of burnup was conducted, 
and it showed that the best neutronic performances are achieved with total burnup 29\% FIMA. On the other hand, considering that the total salt inventory is minimized at high burnup, the optimal burnup is a compromise between good performances and high fuel utilization. Fig. 4 shows that the effective multiplication factor of the system decreases with decreasing reactor outer radius.

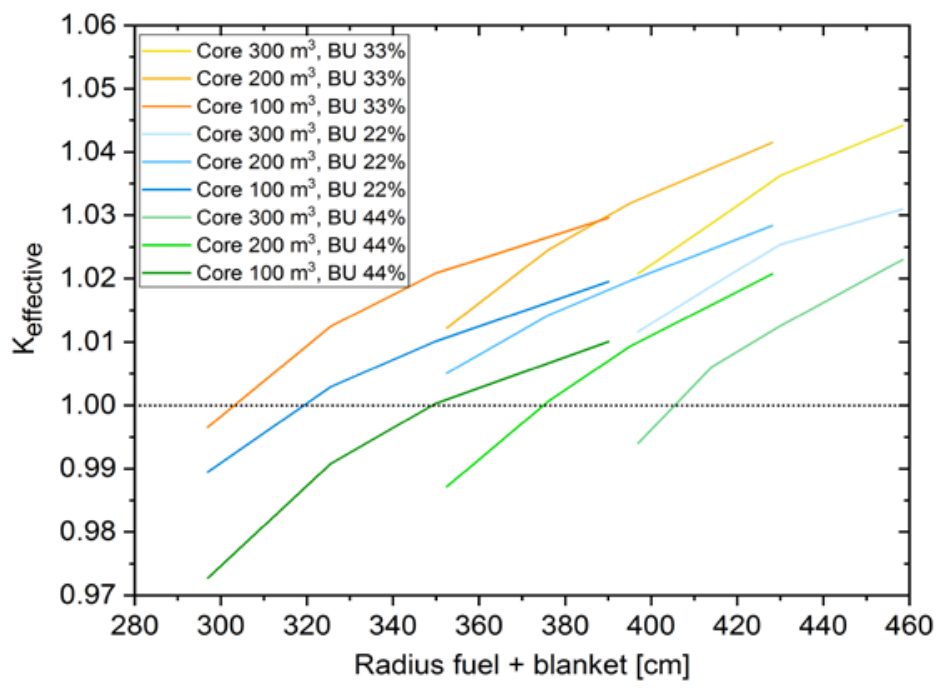

Figure 4. Blanket optimization. Neutron multiplication factor of reactors with different core volumes, blanket volumes and total burnup.

Additionally, it can be noticed that between two combinations with the same radius (thus volume), the one with the bigger blanket is the better performing. This can be clearly seen in Figure 5, where the dependency of $k_{\text {effective }}$ on the fuel and blanket volume is explicitly represented. The blanket burnup resulting from all the geometries studied ranges from $0.2 \%$ to $18 \%$. Within this interval the $k_{\text {infinite }}$ of the blanket, shown in Fig. 5, as well as the atomic density ratio $\frac{{ }^{239} \mathrm{Pu}}{{ }^{238} U{ }^{240} \mathrm{Pu}}$, grows with burnup. Therefore, a higher burnup is associated with a higher concentration of fissile material. At the same time, the blanket burnup increases with the blanket volume: the residence time of the salt in the blanket is proportional to the number of cycles it takes to replace the whole volume. Therefore, in the simulated butnup range, a large blanket guarantees better neutronic performances, allowing the active core size to be decreased. Fig. 5 also shows that the reactivity of smaller cores is more sensitive to variations in the blanket volume. Small cores suffer from a high neutron leakage that is mitigated within the blanket thickness, which is more variable for small cores than for large ones, as shown in Fig. 3.

Overall, the addition of the blanket has a large impact on the performance: thanks to the better neutron economy achieved, the total salt volume could be reduced by more than $30 \%$ compared to the one-fluid configuration.

\subsection{Multi-fluid reactor}

The radial neutron flux distribution could be further optimized by introducing a multi-fluid layout. Three configurations were compared: these have the same active core volume $\left(100 \mathrm{~m}^{3}\right)$ and total blanket volume $\left(100 \mathrm{~m}^{3}\right)$, divided into one, two or three blankets. Refueling is always taking place moving salt from an outer blanket to an inner one. Increasing the number of blankets, the fuel utilization in the core region increases. The radial flux, shown in Fig. 6, peaks more prominently in the center and decays faster in the 
periphery. The peaking of the flux is beneficial in the transition from one to two blankets, in which case the core reactivity increases by approximately $100 \mathrm{pcm}$. In the three blankets case the total leakage is slightly reduced as well. However, the neutron captures of the Hastelloy vessels, which separates one salt layer from the other, increase by approximately $40 \%$ in the three blankets scenario. As a consequence, the whole core reactivity decreases by roughly $200 \mathrm{pcm}$. Overall, higher precision simulations and more detailed parametric study should be done to confirm the optimal multi-fluid design.
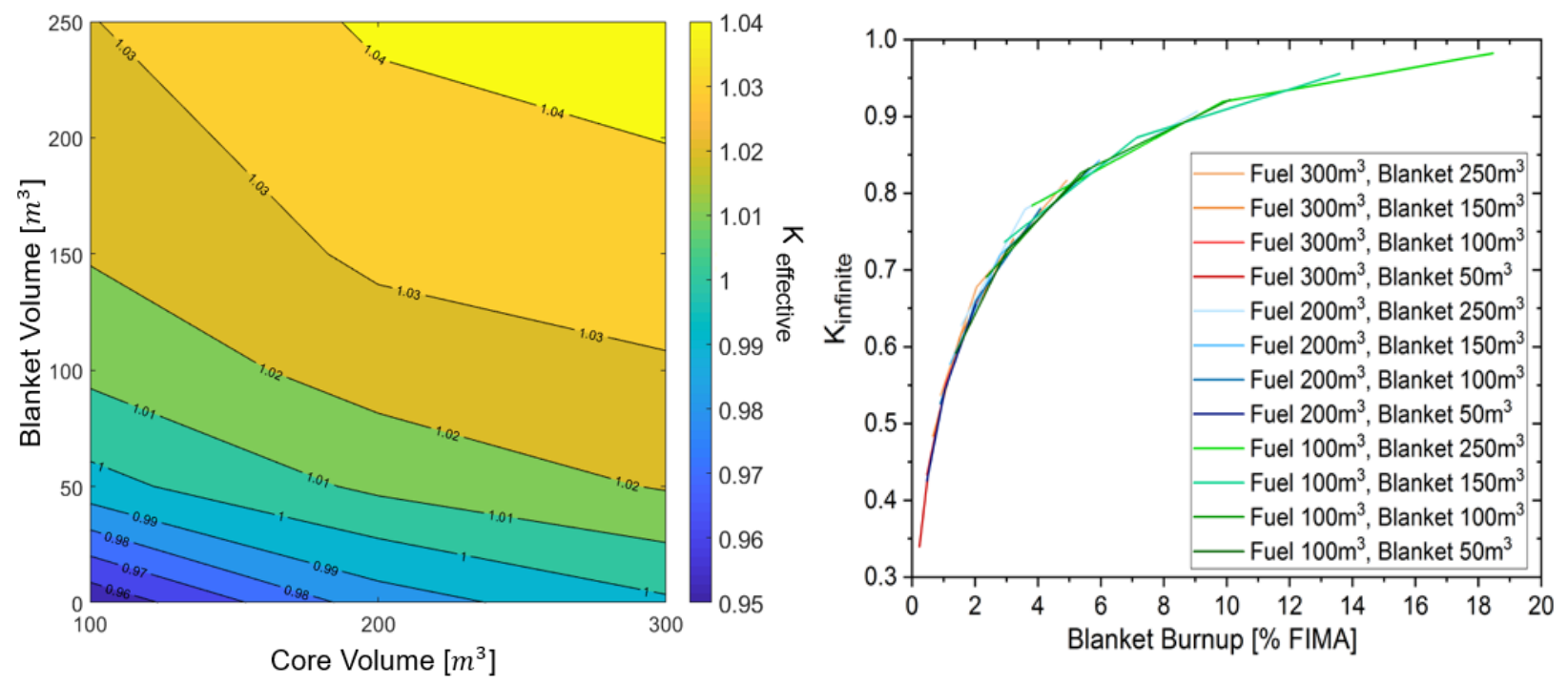

Figure 5. $\mathbf{K}_{\text {effective }}$ and blanket $\mathbf{K}_{\text {infinite. }}$. Dependence of $k_{\text {effective }}$ on the core and blanket volumes for $33 \%$ burnup on the left. Dependence of blanket $k_{\text {infinite }}$ on blanket burnup on the right.

The effect of two moderating materials, graphite and Hastelloy, has been studied in the two-blanket layout. Three moderating shells were introduced in the most external blanket. Moderation increases scattering reducing neutron migration length, and thus leakage. On the other hand, a softer spectrum increases parasitic captures in structural materials and in the salt, modifying the equilibrium composition, i.e. ${ }^{239} \mathrm{Pu} /{ }^{240} \mathrm{Pu}$ [9]. The introduction of Hastelloy increased notably the captures of the system, generating a total reactivity decrease of $940 \mathrm{pcm}$. The design moderated with graphite shows less leakage than the unmoderated case. However, reduced leakage is not sufficient to compensate for the additional losses due to the higher captures in a softer spectrum, which caused a reactivity drop of $350 \mathrm{pcm}$.

\section{CONCLUSIONS}

A Breed and Burn Chloride fueled Molten Salt Reactor was simulated. The feasibility of the B\&B fuel cycle was proven in an infinite core, where different salt compositions and non-soluble fission products removal strategies were investigated. The results show that Uranium fuel salt performs better than Thorium and mixed Uranium Thorium fuel salt in this fuel cycle operation, and that removal of non-soluble fission products is strictly necessary for operation. Afterwards, a design based on a cylindrical geometry was optimized. The target of the optimization was to achieve the smallest volume possible with the highest fuel burnup. Several multi-fluid layouts, composed of an active core and up to three breeding blankets, were proposed. The introduction of the first blanket has a large impact on the performance of the reactor, and it allows the total salt volume to be reduced by more than $30 \%$ compared to the design without blanket. The reactor benefits slightly from the addition of a second blanket, whereas the captures from the structural materials prevail over the reduced leakage in the three-blanket layout. A moderating material was introduced in the most external blanket to reduce neutrons' migration length, but also in this case the 
parasitic captures introduced dominate. Further optimization strategies, like changing the refueling order of the blankets, could be adopted in the future.
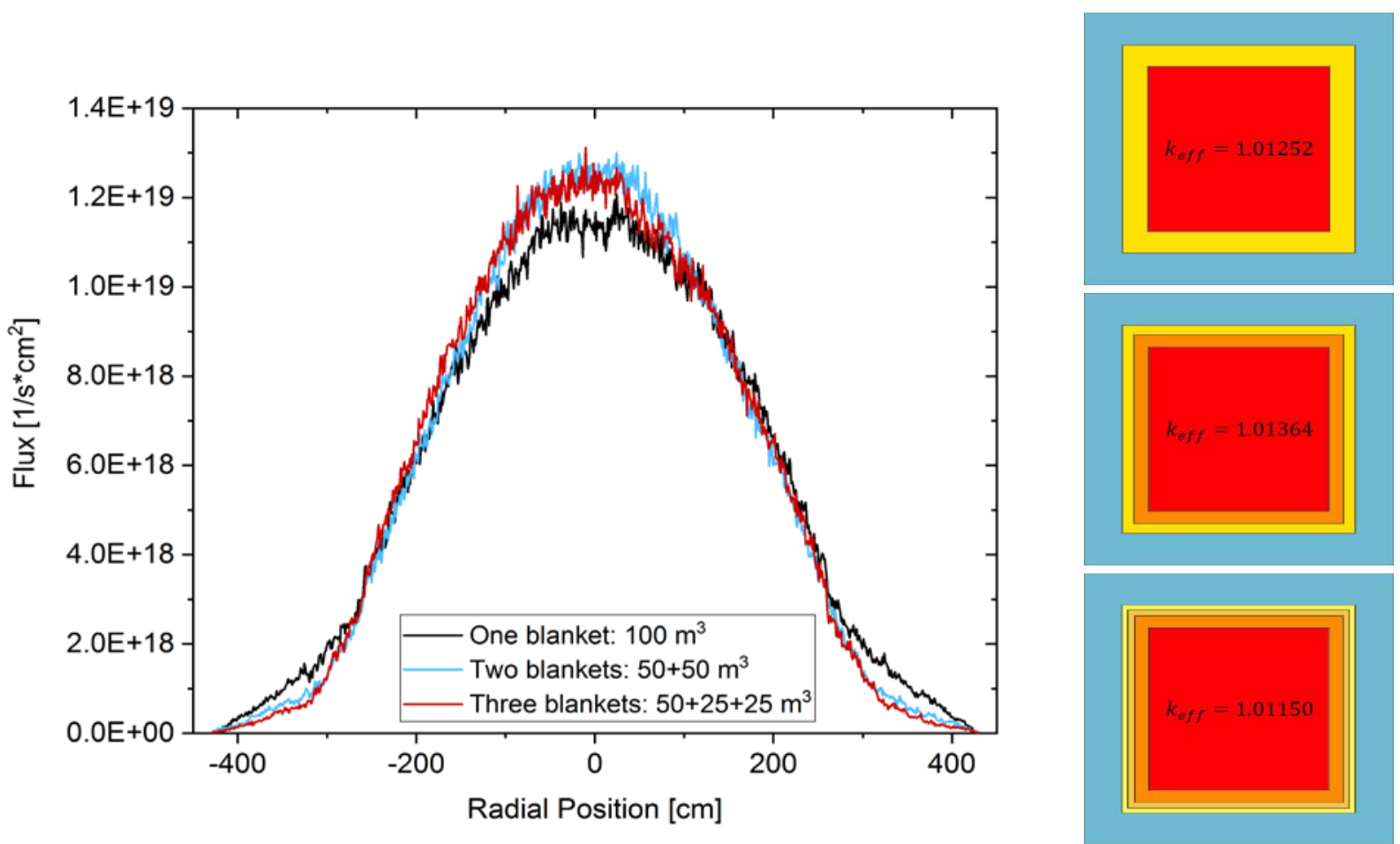

Figure 6. Multi-fluid layouts. Flux distribution and $\mathrm{k}_{\text {effective }}$ in the cases with one, two and three blankets. In the representation on the right, the active core is in red while the lead reflector is in blue.

\section{REFERENCES}

1. Krepel J, Losa E. Closed U-Pu and Th-U cycle in sixteen selected reactors: Comparison of major equilibrium features. Annals of Nuclear Energy. 2019;vol: 128; pp: 341-57.

2. Tomic G, Krepel J. ESFR-SMART core burnup calculation on radially infinite lattice with MonteCarlo code. Proceedings of ICAPP 2019, Juan-les-pins, France.

3. Hombourger B, Krepel J, Mikityuk K, Pautz A. Fuel Cycle Analysis of a Molten Salt Reactor for Breed-and-Burn Mode. Proceedings of ICAPP 2015; pp: 910-6.

4. Martin MV, Aufiero M, Greenspan E, Fratoni M. Feasibility of a Breed-and-Burn Molten Salt Reactor. In: Transactions of the American Nuclear Society, 2017; vol: 116.

5. Hombourger BA. Conceptual Design of a Sustainable Waste Burning Molten Salt Reactor. Doctoral Thesis, EPFL, Lausanne, Switzerlan, 2018.

6. Hombourger B. Breed-and-Burn Fuel Cycle in Molten Salt Reactors. European Physical Journal Nuclear Sciences and Technologies, 2019; pp:1-19.

7. Aufiero M, Cammi A, Fiorina C, Leppänen J, Luzzi L, Ricotti ME. An extended version of the SERPENT-2 code to investigate fuel burn-up and core material evolution of the Molten Salt Fast Reactor. Journal of Nuclear Materials 2013; vol: 441 (1-3); pp: 473-86.

8. De Oliveira R, Hombourger BA. Fuel tap: a simplified breed and burn MSR. In: PHYSOR2020, Cambridge, United Kingdom.

9. Křepel J, Hombourger B, Fiorina C, Mikityuk K, Rohde U, Kliem S, et al. Fuel cycle advantages and dynamics features of liquid fueled MSR. Annals of Nuclear Energy, 2014; vol: 64; pp:380-97. 